\title{
Gangrène du pénis secondaire à une strangulation par un anneau métallique
}

\author{
Zakaria DAHAMI, Ouassil SAGHIR, Abdessamad ELHAOUS, Faical BARJANI, Mohamed GABSI, \\ Mohamed S. MOUDOUNI, Ismail SARF
}

Service d'Urologie, Hôpital Ibn Tofail, CHU Mohamed VI, Marrakech, Maroc

\section{RESUME}

La strangulation du pénis est une pathologie rare nécessitant une prise en charge en urgence. Plusieurs objets, métalliques ou non, peuvent être placés sur le pénis pour augmenter les performances sexuelles ou dans des intentions auto-érotiques.

Nous rapportons l'observation d'un patient schizophrène âgé de $\mathbf{4 4}$ ans qui s'est présenté au service des urgences pour une strangulation du pénis secondaire à un anneau métallique placé à la base du pénis 8 jours avant la consultation, sans trouble urinaire. Une décompression chirurgicale a été indiquée.

Une revue de la littérature nous a permis de mettre en exergue les différents tableaux cliniques de la strangulation du pénis et les méthodes thérapeutiques.

Mots clés : pénis, anneau métallique, strangulation

\section{INTRODUCTION}

La strangulation du pénis par un objet métallique ou non est un traumatisme rare nécessitant un traitement urgent car elle engage le pronostic fonctionnel $[9,13]$.

Depuis 1755 , plusieurs cas ont été rapportés dans le monde entier. La plus large série date de 1948, publiée par Dakin aux Etats Unis [11]. L'âge des patients varie entre 15 et 56 ans. Les objets utilisés le plus souvent sont des anneaux de mariages, des écrous ou des pignons en acier, des bouteilles en plastique, dont le but est de maintenir une érection prolongée afin d'améliorer les performances sexuelles ou parfois à la suite de troubles psychiatriques $[3,4,9,14,16]$.

L'extraction de ces objets représente un grand stress pour le patient et un véritable défi pour le praticien [8]. Les objets non métalliques sont en général faciles à extraire, par contre ceux en acier nécessitent le recours à un matériel de découpage spécial (scie à main ou électrique, pinces à métaux, pinces à air comprimé) qui ne sont souvent pas disponibles dans le service des urgences [15]. Une chirurgie de décompression peut être indiquée en cas d'échec des tentatives sus décrites.

Nous rapportons le cas d'une gangrène de la verge secondaire à une strangulation pénienne par un pignon métallique ayant nécessité une approche chirurgicale.

\section{OBSERVATION}

Monsieur B. F. âgé de 44 ans, célibataire, schizophrène sous traitement neuroleptique (chlorpromazine, halopéridol et trihexyphénidyle) consulte au service des urgences pour une strangulation de la verge par un pignon métallique impossible à extraire, placé au niveau de la base de la verge 8 jours auparavant dans un but d'auto stimulation érotique.

L'examen clinique retrouve une verge en érection, étranglée par un pignon en acier de $3 \mathrm{~cm}$ d'épaisseur,

Correspondance :

Dr. Zakaria DAHAMI - Boulevard Hassan II, résidence Asbahani 1, $n^{\circ} 17$, Gueliz Marrakech, 40 000, Maroc Tel 0021244422409 - Fax 0021222267231 -

Email zdahami@hotmail.com - zdahami@gmail.com 
placé au niveau de la racine avec un important œdème distal, et une nécrose cutanée au niveau du fourreau pénien (Figure 1). Le gland est engorgé, cyanosé, avec une perte totale de la sensibilité. On note par ailleurs l'absence de fistule urétrale ou de troubles mictionnels associés à type de rétention d'urine.

Une tentative pour couper l'anneau par une pince à métaux se solde par un échec vu l'importance de son épaisseur et sa grande résistance. Un traitement chirurgical de décompression sous rachianesthésie est préconisé. L'intervention consiste en une incision cutanée sur la base de la verge, avec dénudation totale du corps caverneux et excision de la totalité du fourreau pénien nécrosé et œdématié dans un premier temps permettant ainsi l'extraction du pignon de la verge sans difficulté (Figure 2).

Le patient évolue bien sous antibiothérapie antistaphylococcique et soins locaux et est adressé à J10 de son hospitalisation en psychiatrie pour prise en charge. L'évolution est marquée 45 jours plus tard par une tentative d'autolyse à l'acide chlorhydrique se soldant par le décès du patient.

\section{DISCUSSION}

La strangulation pénienne par un anneau métallique est un traumatisme grave mais rare qui peut entrer dans le cadre des automutilations s'il est réalisé dans un contexte de troubles psychiatriques avec auto agressivité [13], comme il peut être un simple accident s'il est utilisé pour améliorer la fonction érectile ou comme méthode d'auto-érotisme [3, 4, 14, 16]. Dans tous les cas il requiert une décompression en urgence. Le retour veineux et lymphatique est interrompu en premier, engendrant l'œdème qui apparaît au bout de quelques heures et gène l'extraction de l'anneau. Si la compression évolue, le flux artériel peut être compromis $[11,14]$.

Plusieurs complications possibles de la strangulation peuvent survenir : la rétention urinaire dans $50 \%$ des cas, les fistules uréthrales, le priapisme, l'hypoesthésie distale, l'ulcération et la nécrose cutanées pouvant évoluer vers la gangrène voire l'amputation de la verge. Ces complications dépendent de la durée et de la gravité de la compression $[1-3,8,9,18]$.

Le principe du traitement est simple : c'est la décompression rapide de la verge afin de permettre une bonne vascularisation des tissus. Par contre le choix de la méthode thérapeutique est un véritable challenge pour l'urologue vu la grande panoplie des anneaux utilisés et la variabilité des lésions [14].

Pour faciliter la décision thérapeutique, Bhat et al. ont mis au point une classification simple des traumatismes par strangulation de la verge [3] :

- Grade 1 : œdème distal isolé.

- Grade II : traumatisme uréthral et cutané, compression du corps spongieux, hypoesthésie distale.

- Grade III : traumatisme urétral et cutané avec perte de la sensibilité distale.

- Grade IV : rupture du corps spongieux et/ou fistule urétrale, compression du corps caverneux, anesthésie distale.

- Grade $V$ : gangrène, nécrose ou amputation distale de la verge.

Plusieurs variétés thérapeutiques sont proposées pour prendre en charge ce type de lésions mais le choix reste difficile vu le caractère inhabituel de ce traumatisme. Ce choix peut être orienté par le grade des lésions [11]. De nombreux auteurs ont rapporté des échecs avant de trouver une solution $[1,4,5]$. La première étape est le soulagement de la rétention urinaire par un sondage vésicale pour les grades l et II [10], tandis qu'une cystostomie à minima sera préférable pour les grades supérieurs $[2,7]$.

Les principales techniques à visées thérapeutiques sont regroupées en 5 catégories [11] :

\section{La méthode du fil}

Elle consiste à utiliser un fil de soie ou une bande en latex pour comprimer la zone œdématiée, ce qui facilite le glissement de l'anneau. Elle peut aussi être associée à une aspiration du sang par le gland (Figure 3) [6]. Cette technique donne de bons résultats pour les grades I, II et III, permettant une décompression sans lésions des tissus $[5,12,19]$.

\section{L'aspiration}

On utilise des aiguilles pour aspirer le sang du gland et des corps caverneux ou pour faire des ponctions sous cutanées afin d'évacuer la lymphe qui cause l'œdème. Seuls trois cas d'aspiration sont rapportés dans la littérature pour des strangulations de moins de $14 \mathrm{~h}$, de grades II et III $[3,11,17]$.

\section{Le découpage de l'anneau}

II dépend de l'épaisseur et des matériaux qui constituent l'anneau. Cette méthode est surtout utilisée pour les grades I à III [2]. Elle nécessite un matériel allant d'une simple pince à main à une scie à air comprimée, ce qui n'est pas toujours disponible dans les services d'urologie [15]. 


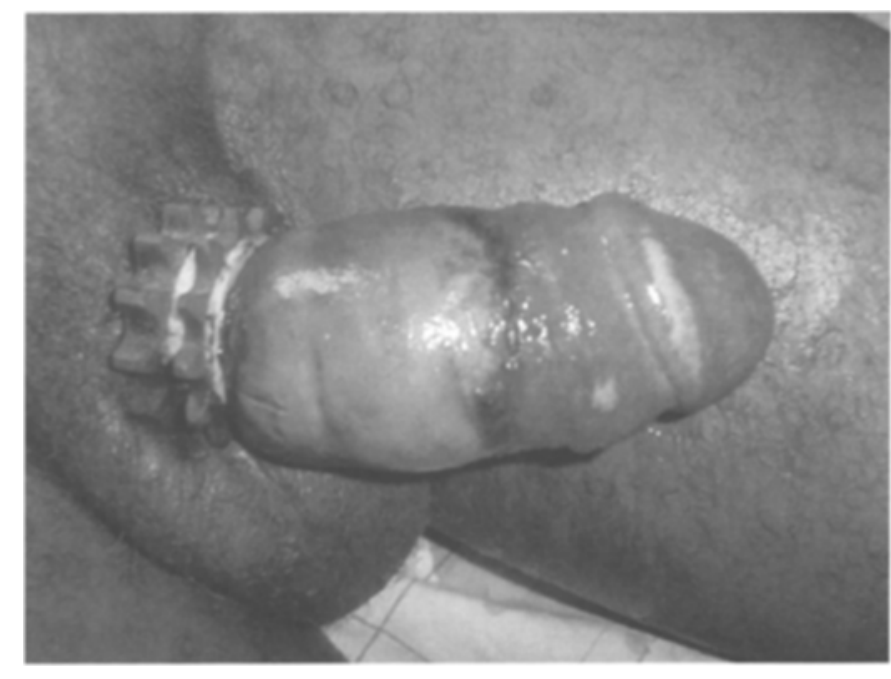

Figure 1: Gangrène de la verge sur pignon placé au niveau de la base du pénis.

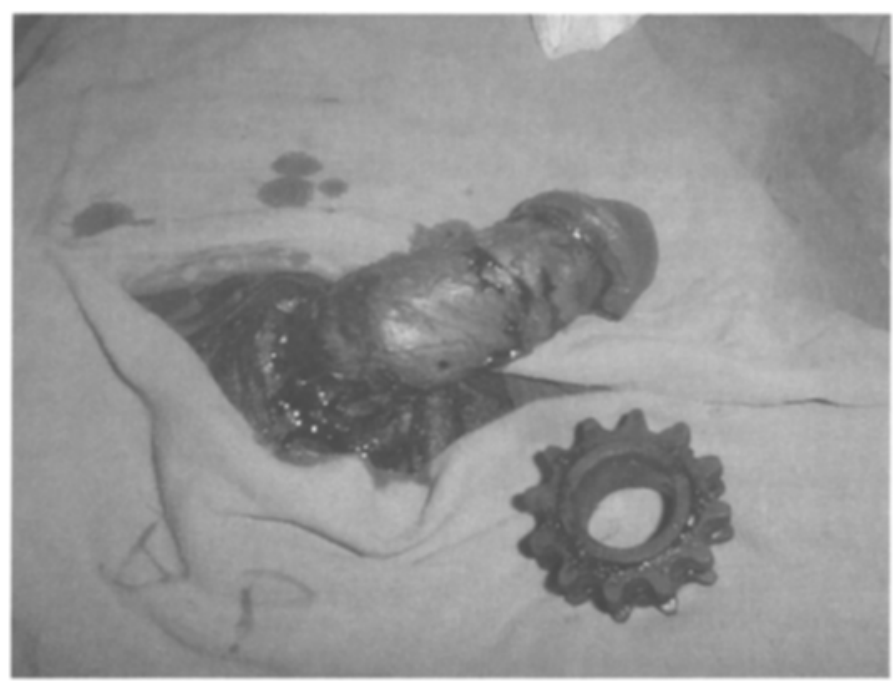

Figure 2 : Excision de la peau pénienne et extraction de l'anneau.

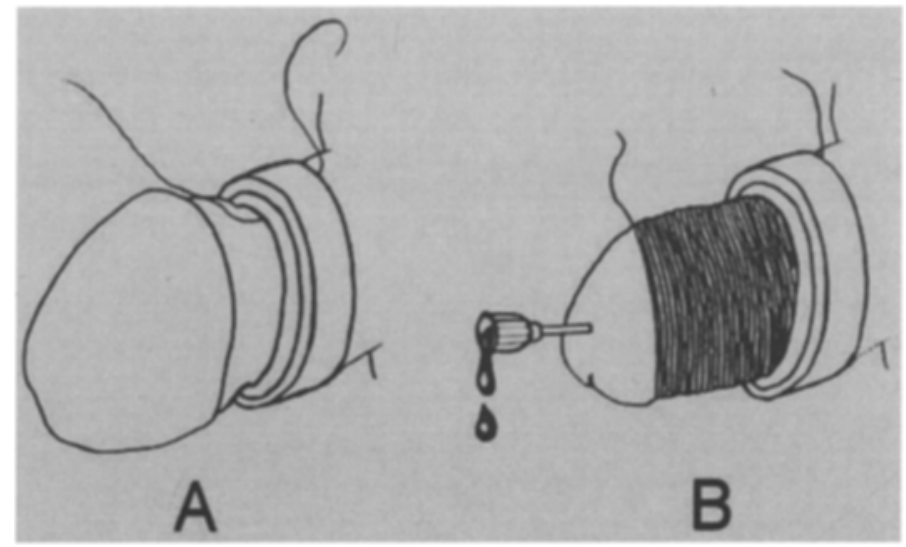

Figure 3 : Méthode de la ficelle modifiée.

A : Passage de la ficelle de soie sous l'anneau.

$B$ : Evacuation de sang noirâtre de façon continue à travers une aiguille, La ficelle de soie est enroulée le long de la verge [6]. 


\section{La chirurgie de décompression}

Elle est préconisée surtout pour les grades $V$ et consiste en une dénudation de la verge jusqu'au fascia de Buck suivie d'une greffe de peau $[1,9]$.

\section{L'amputation réimplantation de la verge sous microchirurgie}

Elle est préconisée pour les grades IV et $V$ s'il n'y a pas de récupération de la sensibilité et du pouls distal au doppler au bout d'une semaine post-opératoire $[2,7]$.

Après la levée urgente de l'obstacle, une prise en charge psychiatrique s'impose. La strangulation volontaire de la verge n'est pathognomonique d'aucune affection particulière. La strangulation du pénis par un anneau métallique peut survenir chez un patient psychotique qui relèvera d'une prise en charge psychiatrique systématique. Dans la majorité des cas une stabilisation psychiatrique est obtenue [13]. La strangulation volontaire de la verge peut survenir dans le cadre de l'hystérie, de la schizophrénie comme dans notre observation, de la dépression mélancolique, de l'alcoolisme et dans certaines perversions sexuelles.

Le rôle du psychiatre est de prodiguer le traitement médicamenteux et psychothérapique le mieux adapté à la pathologie d'origine.

\section{CONCLUSION}

La strangulation de la verge est un traumatisme rare qui nécessite une prise en charge dans les premières heures pour un meilleur pronostic fonctionnel. De diagnostic évident, elle pose le problème du choix de la technique thérapeutique la mieux adaptée au grade lésionnel. La prise en charge de ce problème dépend surtout du raisonnement et du bon sens de l'urologue et des moyens dont il dispose.

\section{REFERENCES}

1. BERMUDEZ A. : Incarceracion del pene por objeto metalico pesado. Arch. Esp. Urol., 1998, $51:$ 483-484.

2. BEST J.W., ANGELO J.J., MILLIGAN B. : Complete traumatic amputation of the penis. J. Urol., 1962, 87 : 134-138.

3. BHAT A.L., KUMAR A., MATHUR S.C. et al. : Penile strangulation. Br. J. Urol., 1991, 68 : 618-621.

4. BROWNING W.H., REED D.C. : A method of treatment for incarceration of the penis. J. Urol., 1969, $101: 188-190$.

5. CHAKRABARTY P.B., DAS B.K. : Strangulation of penis by metal ring. Indian Med. Assoc., 1976, $67: 256$.

6. JOONWHAN., TAEK W.K., TAG. H. et al. : Penile strangulation treated with the modified string method. Urology, 2004, 64 : 33-34.
7. KATO Y., KANEKO S., IGUCHI M., KURITA T. : Strangulation of the penis by a ring. Hinyokika kiyo, 1987, $33: 1672-1675$.

8. KIMBER R.M., MELLON J.K. : The role of special cutting equipment and corporeal aspiration in the treatment of penile incarceration with a barbell retaining collar. J. Urol., 2004, 172: 975.

9. KLEIN F.A., SMITH V.S. : Treatment of incarceration of the penis by tempered steel bushings. J. Emerg. Med., 1987, 5: 5-8.

10. DETWEILER M.B., PERKINS D. : Penile incarceration : a wrapping technique. J. Emerg. Med., 2001, $20: 159-161$.

11. DETWEILER M.B. : Penile incarceration with metal objects. A review of procedure choice based on penile trauma grade. Scand. J. Urol. Nephrol., 2001, 35 : 212-217.

12. MOOREVILLE M., MELLER M. : Penile incarceration with barbell retaining ring. J. Urol., 2001, $166: 618$.

13. MOUFID K., JOUALA., DEBBAGHA. et al. : L'automutilation génitale : à propos de 3 cas. Prog . Urol., 2004, 14 : 540-543.

14. PERABO F.G., STEINER G., ALBERS P., MULLER S.C. : Treatment of penile strangulation caused by constricting devices. Urology, 2002, $59: 137$.

15. RANAA., SHARMA N. : Masturbation using metal washers for the treatment of impotence : painful consequences. $\mathrm{Br}$. J. Urol., 1994, $73: 722$.

16. SANTUCCI R.A., DENG D., CARNEY J. : Removal of metal penile foreign body with a widely available emergency-medicalservices provided air-driven grinder. Urology, 2004, 63 : 1183 1184.

17. SINHA B.B. : Penile incarceration by a metallic object. Br. J. Surg., 1988, $75: 33$.

18. SNOY F.I., WAGNER S.A., WOODSIDE J.R. et al. : Management of penile incarceration. Urology, 1984, 24 : 1820.

19. VAHASARJA V.J., HELLSTROM. P.A., SERLO. W. et al. : Treatment of penile incarceration by the string method : 2 case reports. J. Urol., 1993, $149: 372-373$.

Manuscrit reçu : octobre 2006 ; accepté février 2007. 


\begin{abstract}
Gangrene of the penis due to strangulation by a metallic ring

Zakaria DAHAMI, Ouassil SAGHIR, Abdessamad

ELHAOUS, Faical BARJANI, Mohamed GABSI, Mohamed S. MOUDOUNI, Ismail SARF
\end{abstract}

Penile strangulation is a rare injury that requires urgent management. Various non-metallic and metallic objects are placed around the penis to increase sexual performance or for autoerotic purposes.

A 44-year-old male presented to the emergency department with penile strangulation due to metallic rings placed at the base of the penis 8 days prior to the visit. He did not present any problems of urination. The authors review the scientific literature on this unusual condition and its management.

Keys words: penis, penile strangulation, metallic rings 